\title{
The sunlight beam index
}

\section{J Mardaljevic PhD FSLL ${ }^{a}$ and N Roy $\mathrm{MSc}^{b}$}

${ }^{a}$ School of Civil and Building Engineering, Loughborough University, Loughborough, UK

\author{
${ }^{b}$ VELUX A/S, Ådalsvej 99, DK-2970 \\ Hørsholm, Denmark
}

Address for correspondence: Professor John Mardaljevic,

School of Civil and Building Engineering, Loughborough University,

Loughborough, Leicestershire, LE11 3TU, UK

E-mail: j.mardaljevic@lboro.ac.uk

Received 25 October 2015; Revised 17 November 2015; Accepted XX XXX 2015 
This paper describes a fundamental rethinking of the basis for the evaluation of the sunlight potential of spaces. It provides a robust methodology to answer the question: how much sunlight can enter a room? The measure proposed is the cross-sectional area of beam sunlight that passes through a window. The new measure - called the sunlight beam index - is described, and examples are given for a realistic residential dwelling. The sunlight beam index is determined for a full year on a time-step basis (e.g. every 15 minutes), but it can be aggregated into monthly or yearly totals. The annual total provides a single measure for: one window; a group of windows; or, all the windows for an entire dwelling.

\section{Introduction}

It is generally accepted worldwide that all dwellings should have occasional direct sun penetration through at least some of the windows. The same or similar criteria also apply to other categories of buildings, e.g. schools, residential/care homes, hospitals, etc. The guidelines for different countries / locales vary enormously though they tend to have similar key characteristics, e.g. that a window should receive direct sun for a certain period on, say, the equinox. For example, British Standard 8206-2 recommends that:

"the centre of at least one window to a main living room can receive $25 \%$ of annual probable sunlight hours, including at least 5\% of annual probable sunlight hours in the winter months between $21^{\text {st }}$ September and $21^{\text {st }}$ March" $[1]$.

A recent paper by Darula et al. lists the sunlight recommendations for ten European countries [2]. Three of them are reproduced in Table 1 to illustrate the range in the types of recommendations given.

\begin{tabular}{ll} 
Country & Sunlight duration requirements \\
\hline Czech Republic & At least 1.5 hrs on March $1^{\text {st }}$ or balance of sunlight duration \\
& in the period from February $10^{\text {th }}$ to March $21^{\text {st }}$ is at least \\
& 1.5 hrs; solar altitude is at least $5^{\circ}$ \\
Italy & At least 2 hrs of sun per day in the period February $19^{\text {th }}$ to \\
& October $21^{\text {st }}$ \\
Sweden & At least one room or separable part of a room shall have \\
& access to direct sunlight; at least 5 hrs sunlight between 9 am \\
& and 5 pm at spring and autumn equinox
\end{tabular}

Table 1: A selection of recommended sunlight duration requirements from Darula et al. $[2]$

This paper describes a new metric to assess the sunlight beam potential of arbitrarily complex building apertures, typically windows. The rationale for the new model is the need to create an index of sunlight beam potential for buildings that is a faithful measure of that actually experienced. As noted, existing measures of sun exposure/potential are many and various. However, they all possess one or more of the following weaknesses:

a. They consider only certain times of the day and/or year, e.g. one of the equinox conditions. 
b. They either ignore the direction at which the sun is incident on the window, or employ crude switch mechanisms such as the 'dead angle'.

c. They ignore the size of the window.

d. They ignore or cannot adequately account for the shadowing effects of frame bars or window reveals.

e. They ignore or cannot adequately account for shadowing caused by surrounding structures or buildings.

f. The method employed is restricted to idealised geometry or built forms.

g. The evaluation cannot produce a meaningful, aggregate measure for multiple windows and/or an entire dwelling.

h. The evaluation provides no information on the temporal dynamics of possible sun exposure.

The new model is an attempt to overcome all of the above deficiencies. Furthermore, the new model has all of the characteristics desirable for a robust methodology that could serve as the basis for guidelines and standards. A single, unambiguous measure of sunlight beam potential forms the basis of the new method. The evaluation considers all possible hours of the year when direct sunlight may illuminate a window. The paper describes the theoretical basis of the new metric and demonstrates its application to a realistic building model of a residential house.

\section{Rationale for the new approach}

The intention is to quantify the cross-sectional area of beam sunlight that enters an internal space through a glazed aperture, and to provide a meaningful measure of the cumulative annual potential of this occurrence. When sunlight passes through any aperture in a building (usually a window), the beam cross-sectional area can be reduced by four mechanisms:

1. If the angle of incidence $\theta$ is greater than zero, i.e. anything other than normal incidence.

2. The beam is blocked by any external wall/facade reveal depth (occurs whenever $\theta>0)$.

3. The beam is blocked by any internal wall/facade reveal depth (occurs whenever $\theta>0)$.

4. The presence of any additional external obstruction e.g. balcony, neighbouring buildings etc.

The first three off these mechanisms are illustrated in Figure 1. Assume that the beam and the glazed aperture have the same cross-sectional area: $x^{2}$. When the angle of incidence of the beam to the aperture normal is $\theta$, the cross-sectional area of the beam passing through the aperture is $x^{2} \cos \theta$ in the absence of any internal/external reveal depths (i.e. for infinitely thin walls). In the presence of both external and internal reveal depths, the beam area is further reduced by the projecting structures (i.e. reveals) on the outside and inside of the window plane (Figure 1). 


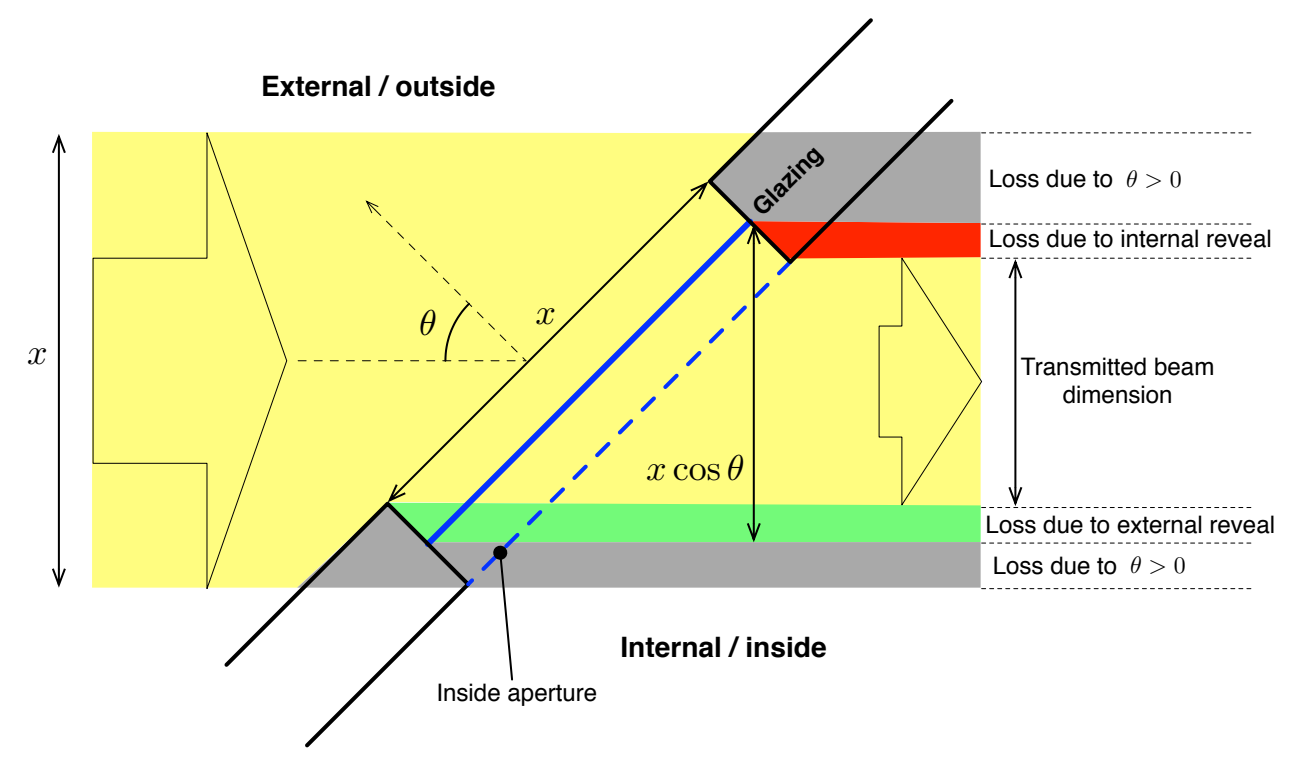

Figure 1: Mechanisms by which beam sunlight cross-sectional area entering a space can be reduced

The preceding made reference to the window plane because this surface is invariably used in existing planning guidelines etc. for the assessment of sunlight availability. Also, building refurbishment could result in an increase to either or both the external and internal reveal depths, e.g. due to added insulation. The position of the glazing plane however is usually unchanged when, say, insulation layers are added. The reduction in beam cross-sectional area due to the internal reveal depth is determined by calculating also the beam sunlight that enters the internal space through a 'virtual' inside aperture (Figure 1).

\subsection{Theoretical Basis}

A single, unambiguous measure of sunlight beam potential forms the basis of the new method. Consider a glazed aperture of area $A_{g}$. When this area of glazing is illuminated by the sun at normal incidence for a period of time $\Delta t$, the sunlight beam index $S_{\Delta t}$ for that duration of time is equal to the product of the illuminated area $A_{i}$ and the time period. Thus the sunlight beam index (or SBI) is a measure of the cross-sectional area and duration of direct sun beam that enters a space. ${ }^{1}$ For the case of normal incidence with no obstructions (i.e. $A_{i}=A_{g}$ ):

$$
S_{\Delta t}=A_{g} \Delta t
$$

However, when the angle of incidence $\theta$ is greater than $0^{\circ}$, the sunlight beam index is reduced by the cosine of the angle because the cross-sectional area of the transmitted beam is reduced by that amount. Thus the illuminance at the window plane serves as a proxy for the reduced cross-sectional area of the transmitted beam:

$$
S_{\Delta t}=A_{g} \cos \theta \Delta t
$$

Most window glass will be fixed in a frame that stands proud 'above' the surface of the glass, i.e. the glass is, in effect, recessed within the frame. Thus, any direct sun

\footnotetext{
${ }^{1}$ Whilst SBI is analogous in nature to any flux-related quantity, there is no actual measure of 'flow' since the beam of sunlight for this purpose is treated as an instantaneous entity.
} 
illumination on the glass at non-normal incidence will result in the frame and/or reveal casting a shadow onto the glass. In other words, the illuminated area of glass will be less than the total area of the glazed aperture, i.e. $A_{i}<A_{g}$. The area of glass in shade (or 'umbra') is $A_{u}$ :

$$
A_{g}=A_{i}+A_{u}
$$

Shading of direct sun can also result from any other structure, nearby (e.g. balcony) or more distant (e.g. obstructing tall building). The angle and obstruction effects are illustrated in Figure 2. Combining the angle and obstruction effects, the generalised
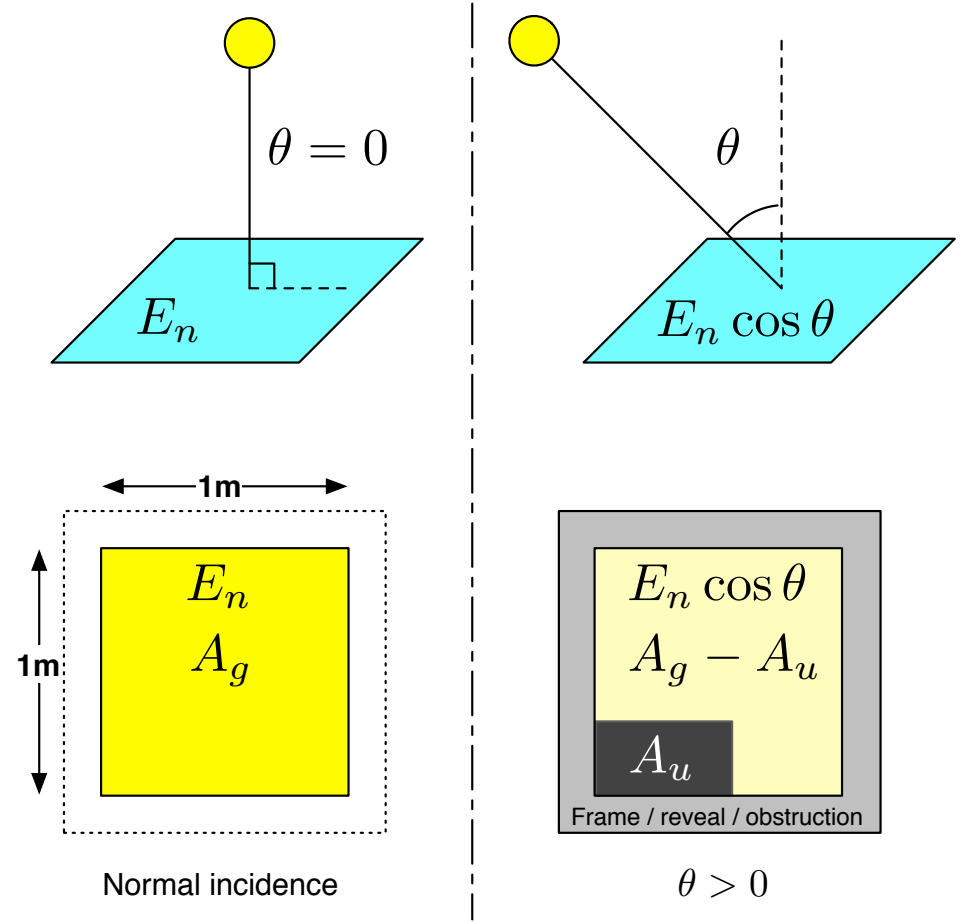

Figure 2: Angle and shading effects in the evaluation of the SBI

sunlight beam index equation is:

$$
S_{\Delta t}=A_{i} \cos \theta \Delta t=\left(A_{g}-A_{u}\right) \cos \theta \Delta t
$$

With the area given in square metres and the time period given in hours (or more typically, a fraction of an hour), the sunlight beam index (SBI) has units of $\mathrm{m}^{2} \mathrm{hrs}$. This formulation makes good sense for a number reasons:

- It is consistent with fundamental illumination physics (e.g. the cosine law of illuminance as a proxy for reduced area of cross-sectional beam).

- The penetration depth of the sun's rays into the space will be reduced with increasing angle of incidence.

- Large incidence angle sun illumination on the window will have a proportionate (i.e. small) contribution in any evaluation without requiring any recourse for arbitrary cut-off conditions, e.g. 'dead angles', etc. 
- The glazed area is properly accounted for.

- Shading - whatever its origin - is properly accounted for.

Any meaningful evaluation must account for the entire year of possible sun positions to capture all of the potential occurrences of sun and and, importantly, shading also. How this is done is described in the following section.

\subsection{Annual sunlight beam Index}

The total sunlight beam index $S_{\text {tot }}$ for any glazed aperture - or group of glazed apertures - is the sum of all the individual sunlight beam indices for the entire year where the sun altitude $\gamma_{s}$ is greater than zero:

$$
S_{t o t}=\sum_{\gamma_{s}>0}\left(A_{i} \cos \theta \Delta t\right)
$$

Additionally, the individual values of $S_{\Delta t}$ for the entire year can be used populate a $2 \mathrm{D}$ matrix that can be visualised as a temporal map $\mathbf{T}$ which has dimensions $24 \times 365$ for hourly data (i.e. $\Delta t=1 \mathrm{hr}$ ):

$$
\mathbf{T}=\left[\begin{array}{cccc}
S_{1,1} & S_{1,2} & \ldots & S_{1,365} \\
S_{2,1} & S_{2,2} & \ldots & S_{2,365} \\
\vdots & \vdots & & \\
S_{24,1} & S_{24,2} & \ldots & S_{24,365}
\end{array}\right]
$$

For a time-step of 15 minutes, the temporal map array has dimensions $96 \times 365$. For a space with $n$ multi-aspect windows (or window groups) labelled $a, b$, etc. the total SBI for the space is simply the sum of the individual SBIs:

$$
S_{\text {space }}=S_{a}+S_{b}+\ldots+S_{n}
$$

Similarly, the temporal map for the space is the sum of the individual temporal map arrays:

$$
\mathbf{T}_{\text {space }}=\mathbf{T}_{a}+\mathbf{T}_{b}+\ldots+\mathbf{T}_{n}
$$

If required, the total SBI for a dwelling or building can be obtained by summing all SBIs for the relevant windows or window groups. Thus it becomes possible to characterise the sunlight beam index for an entire building (e.g. dwelling) with is single SBI value.

The combining of individual temporal map arrays allows for the creation of a visual algebra whereby the temporal dynamics of sunlight beam for an entire building of arbitrary complexity can be readily conveyed to, say, the designer. For example, the temporal maps for individual windows can be incrementally summed (or subtracted) to immediately reveal - and communicate - the effect of design changes/options.

\subsection{Computation of SBI}

The Radiance lighting simulation system was used as the 'engine' in the implementation described here [3]. In fact, the SBI simulation tool used was a subset of a generalised climate-based daylight modelling (CBDM) tool referred to as the ' 4 component' method [4]. Note, however, that lighting simulation per se is not required to compute SBI since the method depends only on a line-of-sight calculation, the modelling of inter-reflection and/or the transmission/scattering effects of light are not needed. Thus, SBI could in 
principle be computed by any 3D CAD/BIM tool that can determine if there is line-ofsite visibility between two points: one in the building model, the other at a sun position on the sky vault.

The accuracy and precision of the computed SBI depends primarily on the faithfulness of the 3D model. In short, good geometry should ensure a reliable result. Simulation parameters which have an influence on the outcome are:

1. the simulation time-step combined with the sun discretisation scheme (these two are related); and,

2. the density of the sensor grid at the window aperture.

The setting of these parameters is essentially arbitrary, incurring only an overhead in computational resources. For the examples shown in this paper a 15 minute time-step was used, i.e. the sun position was determined at 15 minute intervals for an entire year. Potential sun positions on the sky vault at each time step were taken from a pre-determined set of 2,056 evenly distributed points. In the ' 4 component' CBDM tool, these points are used to compute the daylight coefficient matrix for the direct sun component of illumination [4]. The divergence between an actually occurring sun position (at each time step) and the nearest pre-computed point on the sky vault was never greater than $2.3^{\circ}$. This granularity in spatial discretisation for the pre-computed sun positions is comparable with a time-step of approximately 9 minutes, i.e. approximately commensurate with the 15 minute time-step used for the computation of SBI. There would be no advantage with regards to the accuracy of the predicted SBI if there were a significant mis-match between these two time-step values.

The density of the sensor grid at the window aperture was determined by indirect means using an approach which is commonly known as the 'stencil method'. The 'stencil method' is described in the Appendix together with an illustration demonstrating the scalability of the Radiance-based tool to compute SBI.

\section{SBI illustration}

\subsection{One metre square aperture}

Consider a $1 \mathrm{~m}$ by $1 \mathrm{~m}$ square glazed aperture. When this is illuminated at normal incidence by the sun for a period of 1 hour, the sunlight beam index (SBI) for that period is:

$$
1 \times 1=1 \mathrm{~m}^{2} \mathrm{hrs}
$$

In other words, a beam of sunlight with a cross-sectional area of $1 \mathrm{~m}^{2}$ passed through the window aperture for a period of $1 \mathrm{hr}$. If instead the glass were illuminated at an angle of incidence of $60^{\circ}$ for the same $1 \mathrm{hr}$ period, the SBI would be:

$$
1 \times \cos 60 \times 1=0.5 \mathrm{~m}^{2} \mathrm{hrs}
$$

Because, at $60^{\circ}$ incidence the cross-sectional area of the transmitted beam is half what it was at normal incidence. Additionally, the glazed area may be partially shaded by the window reveal and/or external structures. Say, $0.3 \mathrm{~m}^{2}$ of the glazed are is in shade, giving an illuminated area $0.7 \mathrm{~m}^{2}$. The SBI would now be:

$$
0.7 \times \cos 60 \times 1=0.35 \mathrm{~m}^{2} \mathrm{hrs}
$$




\subsection{Annual SBI}

The annual sunlight beam on the $1 \mathrm{~m}^{2}$ window aperture in the vertical plane was predicted using clear sunny skies for a full year. The geographical locale used to generate the sun positions was London (UK), and a time-step of 15 minutes was used, i.e. there were approximately 17,520 above horizon sun positions accounted for in the evaluation. The SBI was simulated for the window in eight orientations: the cardinal and mid-cardinal compass points. This was done for both an unobstructed window aperture and one set in a $20 \mathrm{~cm}$ reveal depth. The results are shown in Table 2. Immediately evident is the expected large variation in SBI with respect to orientation. The aperture reveal depth also has a significant effect, and across all orientations. For example, for the south facing orientation it reduces the SBI from $1,927 \mathrm{~m}^{2} \mathrm{hrs}$ to $1,340 \mathrm{~m}^{2} \mathrm{hrs}$.

\begin{tabular}{lrr}
$\begin{array}{l}\text { Aperture } \\
\text { orientation }\end{array}$ & $\begin{array}{r}\text { Without reveal } \\
\text { SBI }\left[\mathrm{m}^{2} \mathrm{hrs}\right]\end{array}$ & $\begin{array}{r}\text { With } 20 \mathrm{~cm} \text { reveal } \\
\text { SBI }\left[\mathrm{m}^{2} \mathrm{hrs}\right]\end{array}$ \\
\hline North & 204 & 84 \\
NE & 608 & 414 \\
East & 1,348 & 1,037 \\
SE & 1,826 & 1,343 \\
South & 1,927 & 1,340 \\
SW & 1,822 & 1,342 \\
West & 1,345 & 1,033 \\
NW & 604 & 411 \\
\hline
\end{tabular}

Table 2: SBI for $1 \mathrm{~m}^{2}$ glazed aperture without and with $20 \mathrm{~cm}$ external reveal depth

\subsection{Temporal map example: One window}

An example sunlight beam temporal map (TM) is given in Figure 3. This example is for window (glaz01) on the upper story of a residential building. For this building orientation $\left(135^{\circ}\right.$ clockwise rotation from north) the window faces south-east, i.e. also $135^{\circ}$. The building location for this example is Ostersund, Sweden. From the TM the pattern of night (dark grey) and day (light grey or colour) is clearly visible. Daytime light grey indicates that the sun is above the horizon but has no direct visibility of any part of the window area either because the sun is 'behind' the window (i.e. angle of incidence $>90^{\circ}$ ) or it is blocked by local obstructions. Where there is an evident light grey 'notch' in the pattern of colour, this usually indicates that the window is being shaded by some local obstruction (surrounding buildings were present in the model). A yellow shade indicates that sun can be incident on the window at near normal incidence. Whereas a blue shade indicates large angle of incidence direct sun and/or significant obstruction.

The plot title contains the following: the annual total SBI $\left(1,754 \mathrm{~m}^{2} \mathrm{hrs}\right)$; the area of the glazed aperture $\left(1.54 \mathrm{~m}^{2}\right)$; and, the normalised annual total SBI $(1,139 \mathrm{hrs})$. The normalised annual total SBI is simply the annual total SBI divided by the area of the glazed aperture. The normalised annual total can be taken to be an SBI 'efficiency' measure which could be used to make comparison between various window types and/or arrangements. 



Figure 3: Temporal map example

\subsection{Temporal map example: Complete dwelling}

For this illustration, temporal maps were generated for all 16 windows of the house used in the previous example. There are 16 glazing groups for 10 distinct space types, Figure 4. This 'Row House' model is surrounded by neighbouring houses (not shown), and the effect of horizon obstructed by houses in the distance is accounted for by an 'enclosing' cylinder. The combined temporal map for the entire dwelling is shown in Figure 5. The numerical total for SBI across the year was $24,520 \mathrm{~m}^{2} \mathrm{hrs}$ - a single figure can characterise the sunlight beam potential of an entire dwelling.

Eight of the sixteen temporal maps are shown in Figure 6. This figure is best viewed on-screen and enlarged. Note that the false colour scale varies according to glazing area for each window or window group. At a glance one can appreciate the patterns in annual sunlight beam potential for the entire house on a window group by window group basis. This visual presentation of data becomes particularly effective when comparing, say, the temporal maps for the same house design given different orientations. Quite dramatic difference in the patterns of annual SBI are observed for changes in orientation, and the approach would appear well-suited for the evaluation of housing masterplans etc.

\subsection{Effect of internal reveals on SBI}

As noted in Section 2, any non-zero internal reveal depth will lead to a reduction in the cross-sectional area of any beam that passes through the window aperture (for all $\theta>0$ ). The beam sunlight that enters the main volume of the space (i.e. that is not 'lost' in the internal reveal) is determined in the same manner as above, only now an 'inside aperture' 

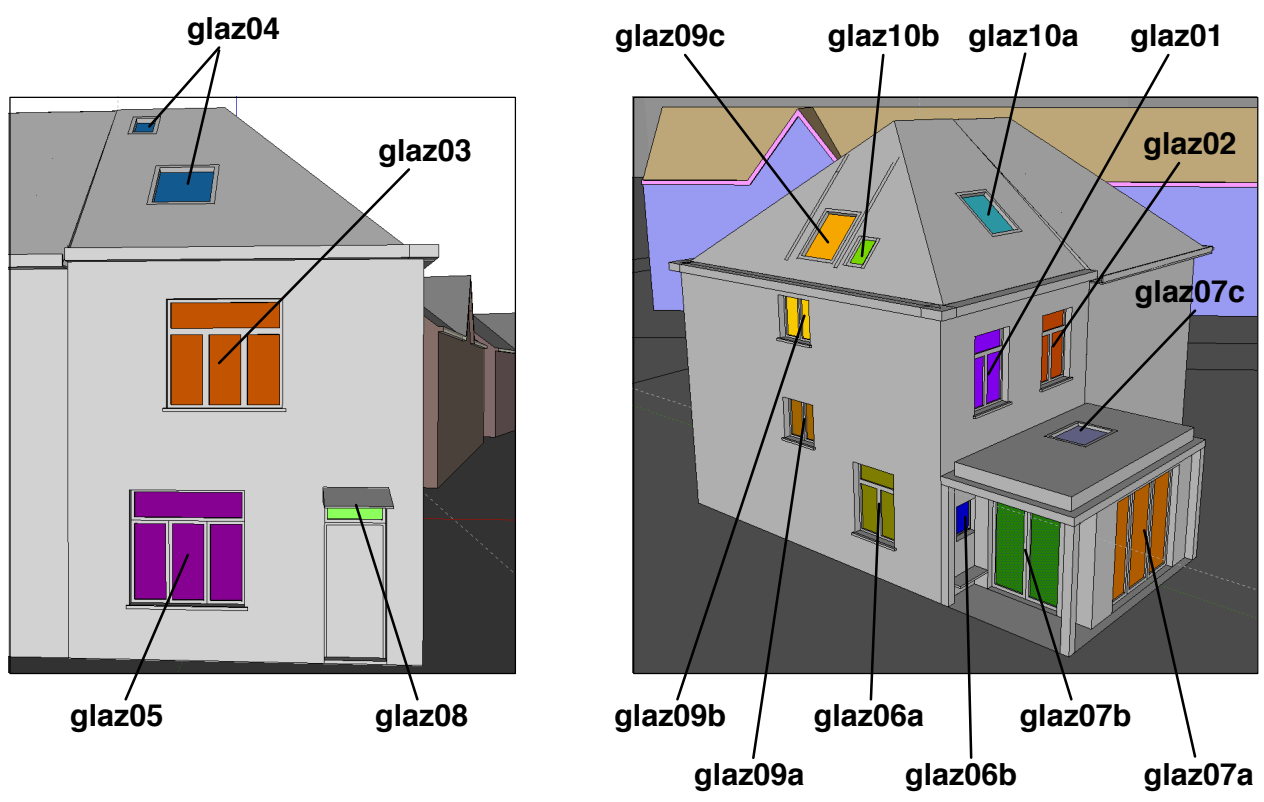

Figure 4: Glazing elements/groups in the 'Row House' model

parallel to the window serves as the calculation grid. The internal aperture is flush with the internal wall boundary and entirely covers the window opening (from this position). In other words, any beam sunlight that passes through the window aperture must pass through the internal aperture in order to enter the space, Figure 7 (see also Figure 1). The internal aperture is often larger that the window due to the presence of the frame. Note that, any window frame in front of the glazing aperture can block beam sunlight in the same way as the external reveal. Similarly, any window frame 'behind' (i.e. inside) the glazing aperture can block beam sunlight the same as the internal reveal.

The effect of internal reveal is demonstrated for two wall thicknesses: $300 \mathrm{~mm}$ and $600 \mathrm{~mm}$ (the latter indicating a very thick, super-insulated wall). In each case, the external glazing surface has a $50 \mathrm{~mm}$ recess (or external reveal), resulting in internal reveal depths of $250 \mathrm{~mm}$ and $550 \mathrm{~mm}$. The glazing area for the model shown in Figure 7 was $1.21 \mathrm{~m}^{2}$ (same for the model with the thicker wall). The glazing orientation was due south and the SBI predicted for a London (UK) location. The results are summarised in Figure 8. The bar chart shows the monthly SBI totals for three internal reveal depths: $000 \mathrm{~mm}$ (i.e. same as that calculated at the glazing aperture); $250 \mathrm{~mm}$; and, $550 \mathrm{~mm}$. The plot is annotated with the annual total for each case. Also shown are the three corresponding temporal maps. From the annual totals, approximately a quarter of the beam sunlight that passes through the window is 'lost' in the $250 \mathrm{~mm}$ deep window reveal, and more than half in the $550 \mathrm{~mm}$ deep reveals. As expected, the blocking effect of the internal reveal is greatest in the summer months when the angle of incidence between the sun and the window normal is greatest.

\subsection{Volumetric assessment of beam sunlight}

For this final part of the exposition of this new approach, the potential to make some meaningful aggregate measure and visualisation of the volume of space 'penetrated' by direct beam sunlight is investigated. For this a very simple space is used: height $\times$ width $\times$ depth was $3 \mathrm{~m} \times 3 \mathrm{~m} \times 5 \mathrm{~m}$, with a $1 \mathrm{~m} \times 1 \mathrm{~m}$ glazed aperture positioned centrally in one of the walls, Figure 9(a). The sensor array is now a 'stack' of 12 sensor grids 

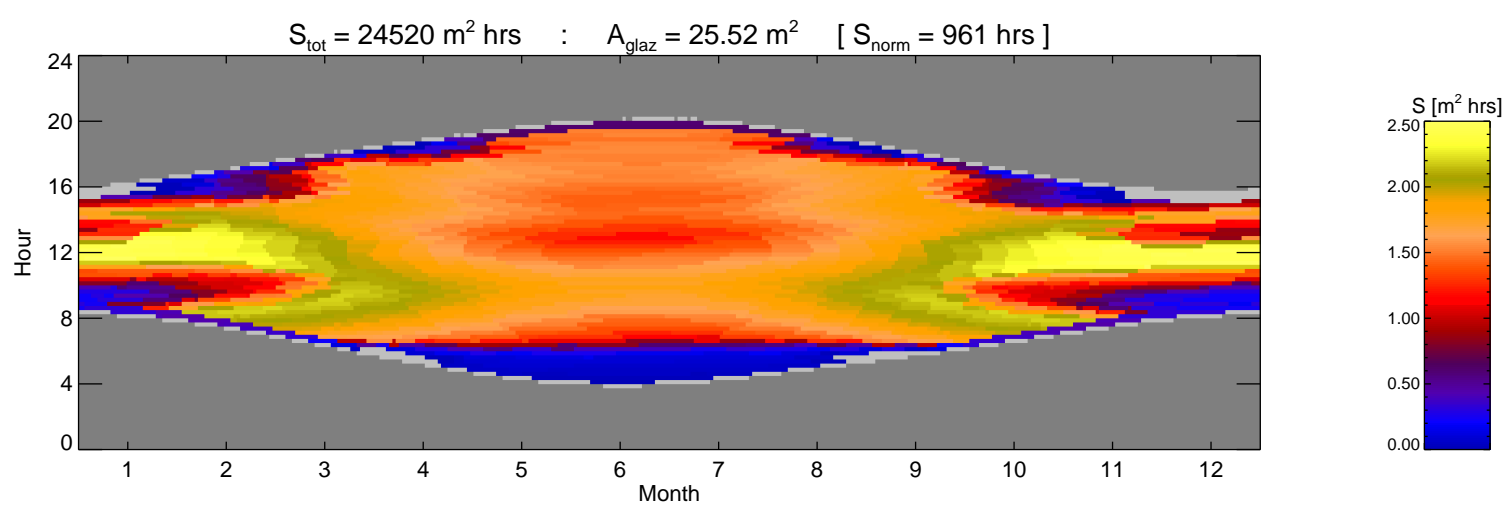

Figure 5: Summed temporal map for the entire dwelling (all 16 window groups)

separated by $10 \mathrm{~cm}$ intervals, and starting from a height of $5 \mathrm{~cm}$ above the floor. Thus the sensor array accounts for a volume of height $1.2 \mathrm{~m}$ above the floor. This was chosen because this is typically the 'occupied' height above the floor for people seated in a space. To be consistent with daylight simulation recommendations, there is a $0.5 \mathrm{~m}$ perimeter space between the sensor array and the walls, i.e. each sensor plane has dimensions $2 \mathrm{~m}$ $\times 4 \mathrm{~m}$ [5]. Each point on the sensor arrays now represents a volume element (or voxel) rather than an area.

The simulation was run as before for a full year at a time-step of 15 minutes and, for this illustration, the location of the room was Ostersund (Sweden) and the window aperture was facing due south. For each time-step $\Delta t$ that sunlight was incident on a sensor array point (voxel volume $v \mathrm{~m}^{3}$ ), the volumetric sunlight beam potential at that point is simply $v \Delta t \mathrm{~m}^{3} \mathrm{hrs}$. The annual total is then the sum of the individual voxel values over the year.

A volumetric rendering of the total annual sunlight beam potential is given in Figure $9(\mathrm{~b})$ - four views of the same volume are given. For this rendering of 3D data, the voxel opacity is proportional to the magnitude of the value at that point. Thus, the very low values (shaded black) are given a very low opacity and so appear as a 'grey haze' allowing the viewer to 'see through' to the shaded higher values (yellow/white). Additionally, it is also possible to determine a single numerical total volumetric potential for beam sunlight - for this case it was $1,785 \mathrm{~m}^{3} \mathrm{hrs}$. It remains to be determined how best to interpret and apply measures of the volumetric potential of beam sunlight in spaces.

\section{Discussion}

This paper has described what is, in effect, a fundamental rethinking of the basis for the assessment and quantification of sunlight potential in spaces. The approach is founded on the long-term quantification of the cross-sectional area of beam sunlight that can enter a space. The approach accounts for all potential losses due to obstructions of any kind and at any scale. Furthermore, the approach distinguishes between losses calculated at the window plane, and those due to the internal construction of the building (e.g. internal sill depth). The graphical/numerical outputs have varying degrees of granularity: the SBI can be presented as a temporal map for one or more windows (i.e. every value for the year at, say, a 15 minute time-step), or aggregated into monthly/annual numerical totals. 


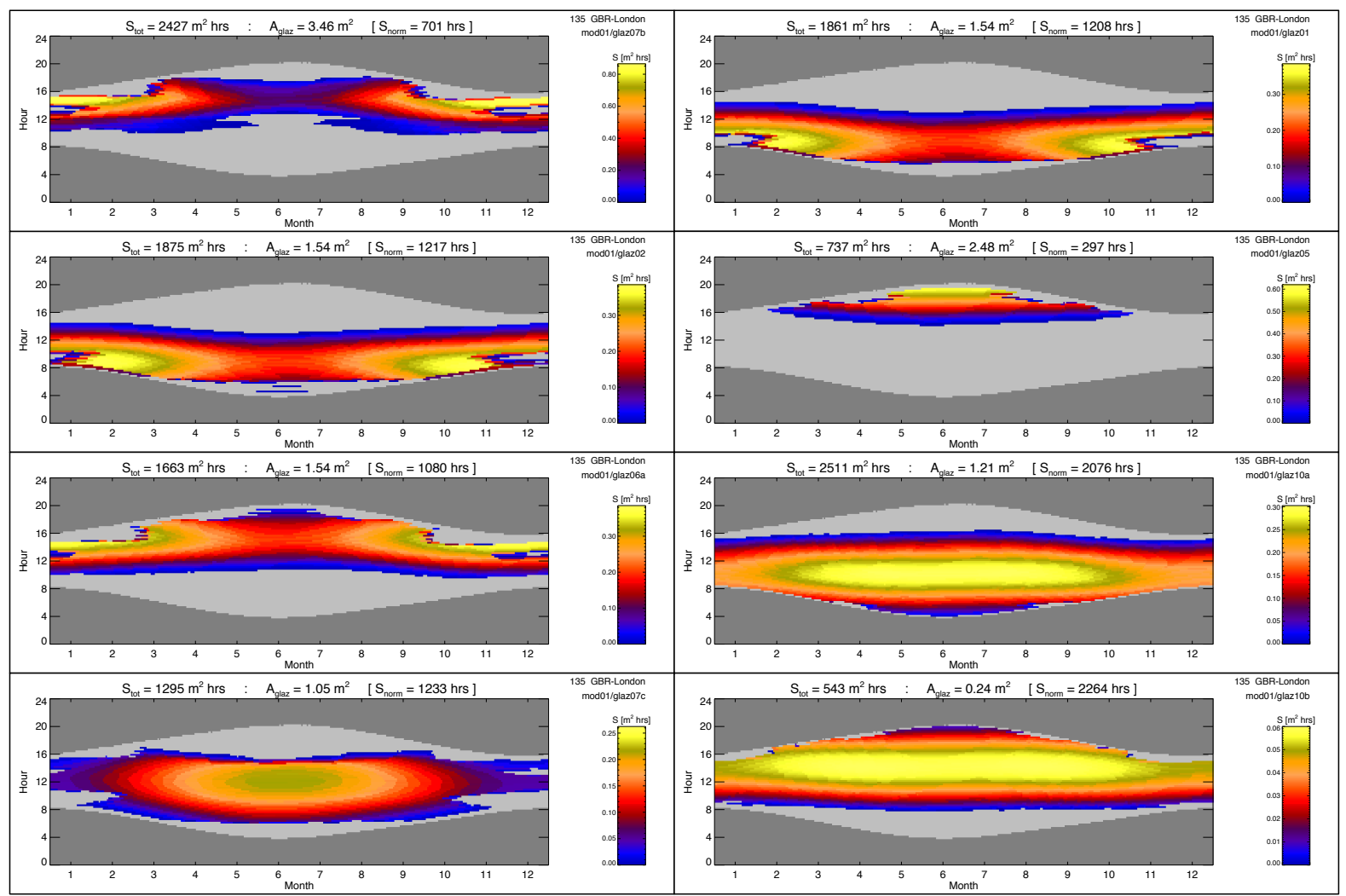

Figure 6: Eight of the sixteen SBI temporal maps for the Row House model

The approach is highly scaleable and can accommodate any practical building geometry constructed using a CAD/BIM system.

The inherent simplicity and scalability of the approach described in this paper indicates that it could form a common basis for the evaluation of sunlight across, say, the EU/CEN countries. Although the existing guidelines vary considerably from one country to the next, the purpose of each is to make some meaningful assessment of sunlight potential [2]. The work described here will be expanded upon to determine its applicability as a basis for future EU/CEN guidelines. This will involve an evaluation of SBI alongside a variety of the measures recommended in current European guidelines/legislation.

No mention has been made thus far regarding glazing transmissivity and its effects on SBI. This is another area to be explored since it would require the evaluation of absolute values for the intensity of the transmitted sunlight beam. This in turn leads to a consideration of the prevailing climate as an indicator of the likely occurrence of sunny conditions throughout the year. To account for the attenuation of SBI due to glazing transmission - including its angular dependency - the sensor grid is placed just 'behind' (instead of just in 'front' of) the glazing. The window is now modelled with the correct transmission properties for the particular glass type. A maintenance factor accounting for reduced transmissivity due to dirt could also be included. In 2003 the World Meteorological Organization (WMO) defined sunshine duration as that period during which the direct solar irradiance exceeds $120 \mathrm{Wm}^{2}$ [6]. Applying local climate data, and using the WMO definition as a threshold, the SBI approach could readily be extended to determine the prevailing intensity of entrant sunlight beam as well as its cross-sectional area over time. It remains to be determined how best such measures could 

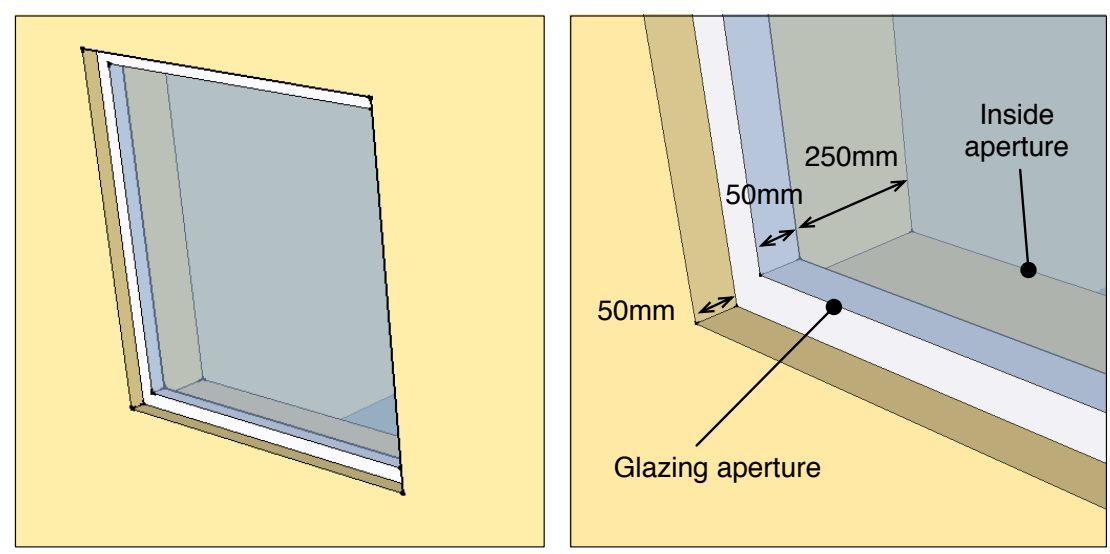

Figure 7: Model showing internal $(250 \mathrm{~mm})$ and external $(50 \mathrm{~mm})$ reveal depths for $300 \mathrm{~mm}$ wall

be used to quantify entrant sunlight as it is experienced by building occupants.

\section{Funding}

The research described in this paper is based on a study commissioned by the VELUX Corporation.

\section{Acknowledgements}

Prof. Mardaljevic acknowledges the support of Loughborough University. Both authors are grateful for the helpful comments received from Dr Jens Christoffersen (VELUX Corporation) and Per Arnold Andersen (VELUX Corporation) during the period of this study and on the drafts of this paper.

\section{References}

[1] BSI. Lighting for buildings. Code of practice for daylighting BS 8206-2. British Standards Institute, 2008.

[2] S. Darula, J. Christoffersen, and M. Malikova. Sunlight and insolation of building interiors. 6th International Building Physics Conference (IBPC), Turin, Italy 14 - 17 June, 2015.

[3] G. Ward Larson, R. Shakespeare, J. Mardaljevic, C. Ehrlich, E. Phillips, and P. ApianBennewitz. Rendering with Radiance: The Art and Science of Lighting Visualization. San Francisco: Morgan Kaufmann, 1998.

[4] J. Mardaljevic. Daylight Simulation: Validation, Sky Models and Daylight Coefficients. PhD thesis, De Montfort University, Leicester, UK, 2000.

[5] LG5 CIBSE/SLL. Lighting Guide 5: Lighting for Education. Chartered Institution of Building Services Engineers, London, 2011.

[6] World Meteorological Organization. Manual on the global observing system. WMONo. 544, Geneva, 2003. 

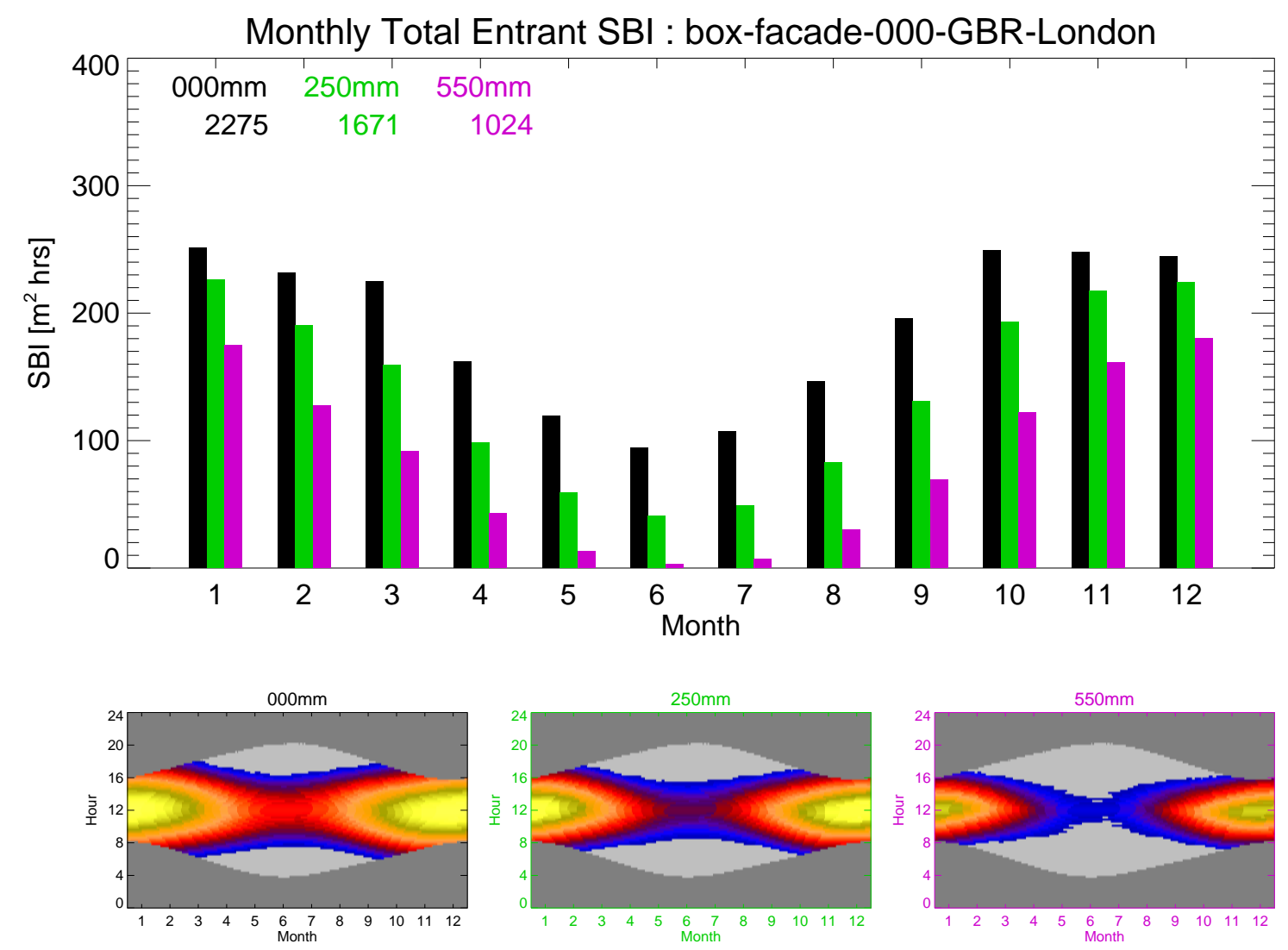

Figure 8: Effect on internal reveal depth on sunlight beam index

\section{Appendix}

\section{A Sensor grid view parameters}

The sensor grid of calculation points is generated from a Radiance rendering of the aperture surface, e.g. a window. The image of the sensor plane is generated using the parallel view projection with the viewpoint fractionally offset from the centre. By simply setting the sensor plane material to be self luminous, an image of the sensor plane is generated whereby the pixels of the plane have some positive value, and all the other pixels are zero. The $x, y, z$ coordinates of every pixel on the sensor plane can then be derived from the image using a combination of commands from the Radiance toolkit, Figure A1. Thus it is possible to generate sensor grids for arbitrary shaped apertures, and for arbitrary resolutions. For horizontal sensor planes it is a relatively trivial matter to determine the parameters for the parallel projection view file - this can easily be scripted. For nonhorizontal planes it is a little more complicated since there is no straightforward method to generate the view parameters. The method devised for the implementation described here was to simplify the problem by first transforming any arbitrarily orientated surface to the horizontal by applying two rotational transforms. Next, the parallel projection view parameters are determined for the sensor plane in the horizontal position. Lastly, the two rotational transforms are applied to the view parameter file in a reverse sense, and reverse order. The process is described below. 


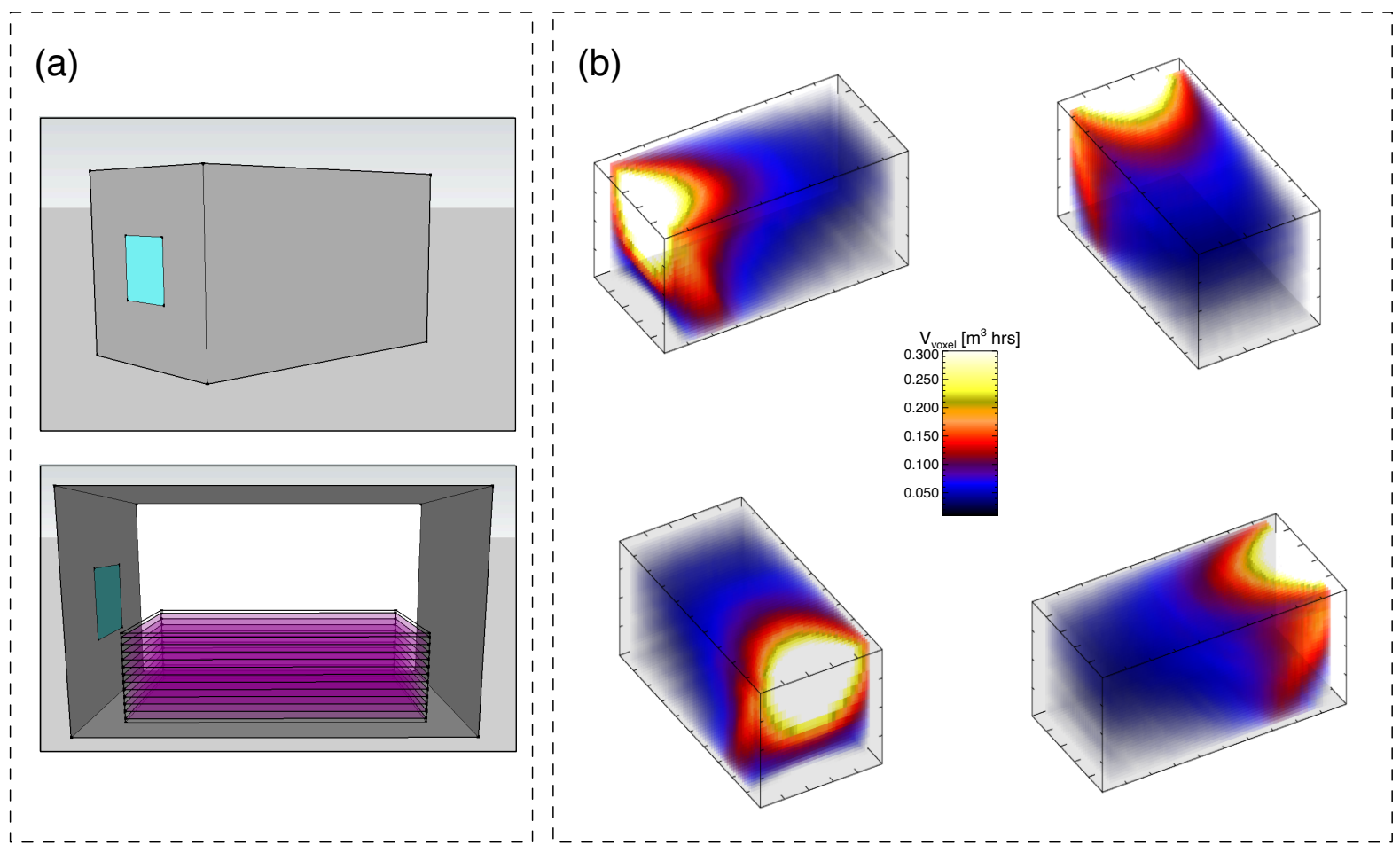

Figure 9: Volumetric display of sunlight beam potential

The vector $\mathbf{n}$ for the (outgoing) normal to any arbitrarily orientated window plane is:

$$
\mathbf{n}=n_{x} \mathbf{i}+n_{y} \mathbf{j}+n_{z} \mathbf{k}
$$

The components $n_{x}, n_{y}, n_{z}$ are determined manually for each (co-planar) window group. Using the Radiance interactive viewer rvu, one glazing element from the group is selected using the new origin command. This creates a new view where the direction is now normal to the selected surface, i.e. the glazing element. This view is then saved to a file so that the view direction vector $n_{x}, n_{y}, n_{z}$ can be extracted for the next stage.

The direction vector is first converted to spherical polar coordinates $\theta$ and $\phi$. The method to generate the correct view file for the window group requires that the window is transformed so that it lies 'flat' in the horizontal plane. This requires two rotations using the xform command:

xform $-r z(90-\phi)-r x \theta$

A script to generate the parallel view parameters needed to create the 'mask' image of the window group is applied to the window in the horizontal plane. Next, the reverse transformation to bring the window group back to the original location is applied to the view parameters for the 'mask' image:

xform $-r x-\theta \quad-r z-(90-\phi)$

Note, the transforms must be applied in the reverse order. Applied in this way, the vectors for the view-point $\mathbf{p}$, view-direction $\mathbf{d}$ and view-up $\mathbf{u}$ (created with the window in the horizontal plane) are correctly transformed to $\mathbf{p}^{t}, \mathbf{d}^{t}$ and $\mathbf{p}^{t}$ for the window in its 


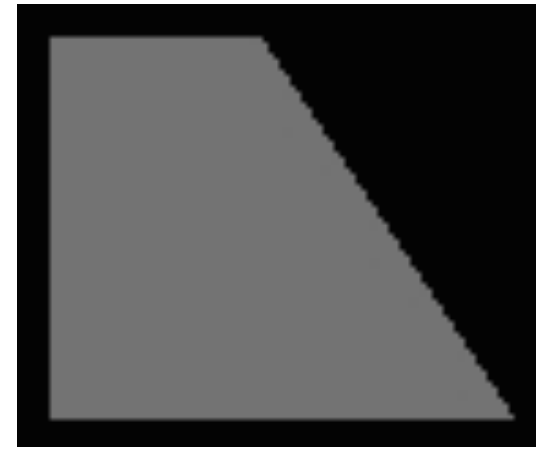

Radiance image of sensor plane

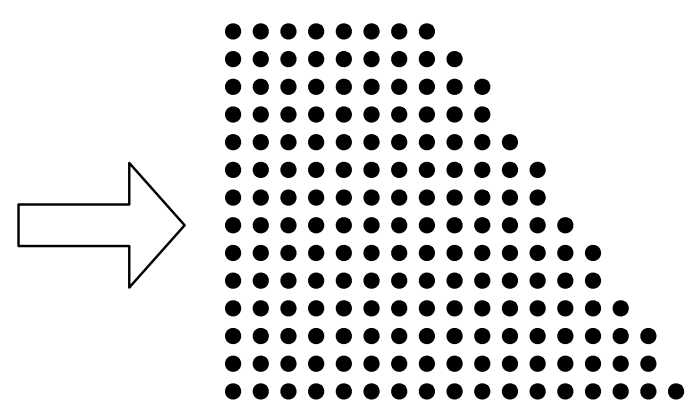

Sensor grid points

Figure A1: Stencil method
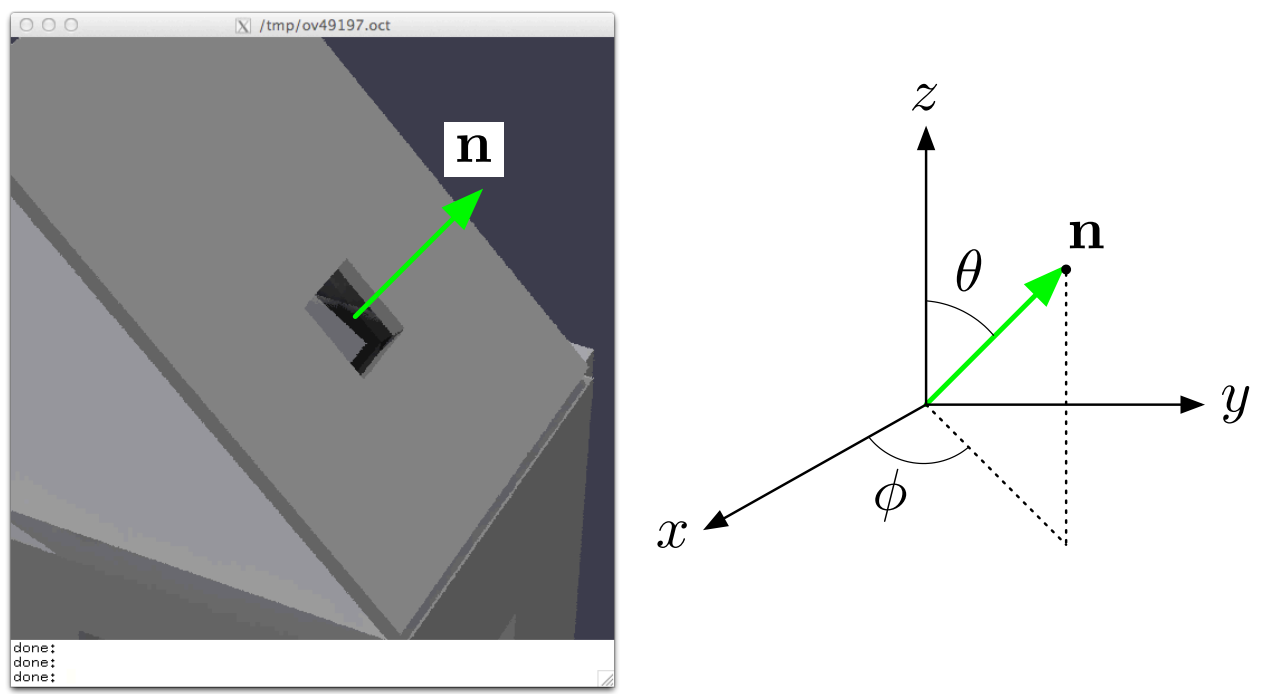

Figure A2: Window transforms

original position, Figure A3.

\section{B Scaleability}

For any practical implementation to have widespread applicability the computational engine should be able to accommodate real-world geometrical complexity, e.g. dense urban environments. As already noted, lighting simulation per se is not required for the computation of SBI. However, the Radiance lighting simulation system is highly regarded for its ability to accommodate building models with a high degree of geometrical complexity whatever the mode of calculation, e.g. direct sun only or full CBDM. This is demonstrated in the following illustration where the residential dwelling model (Figure 4) was 'dropped' into a highly detailed 3D model of New York, Figure A4. A set of SBI values for the dwelling (not shown) were computed in exactly the same manner as before. The only difference being a longer computational time to predict the direct sun daylight coefficient matrices for this, now, much more detailed building model. Scaleability should be an important consideration for any practical implementation to compute SBI since, with the advent of BIM, 3D building descriptions are becoming ever more detailed and complex. 


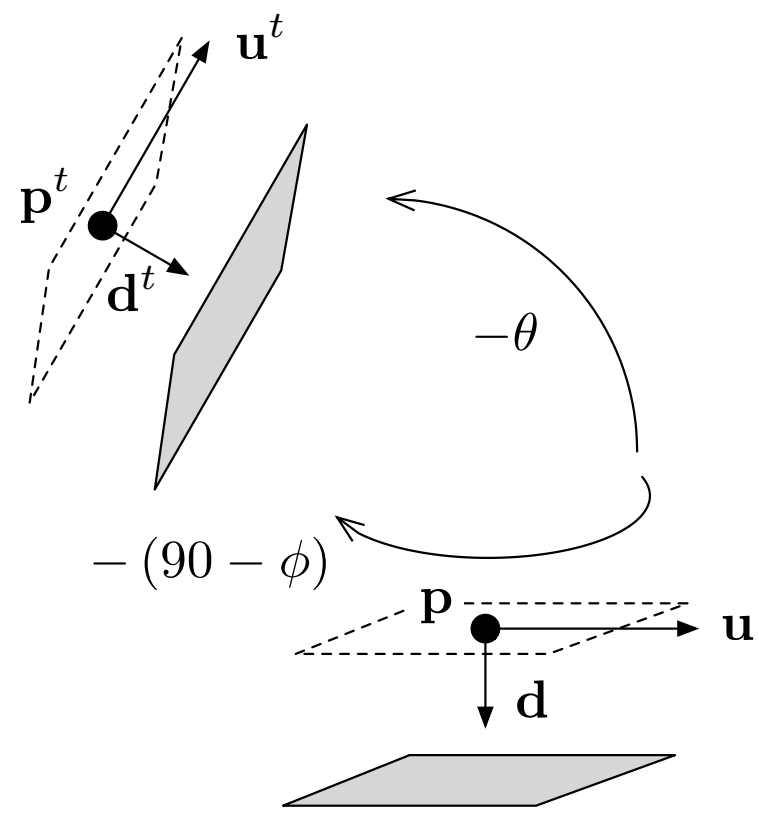

Figure A3: View transforms

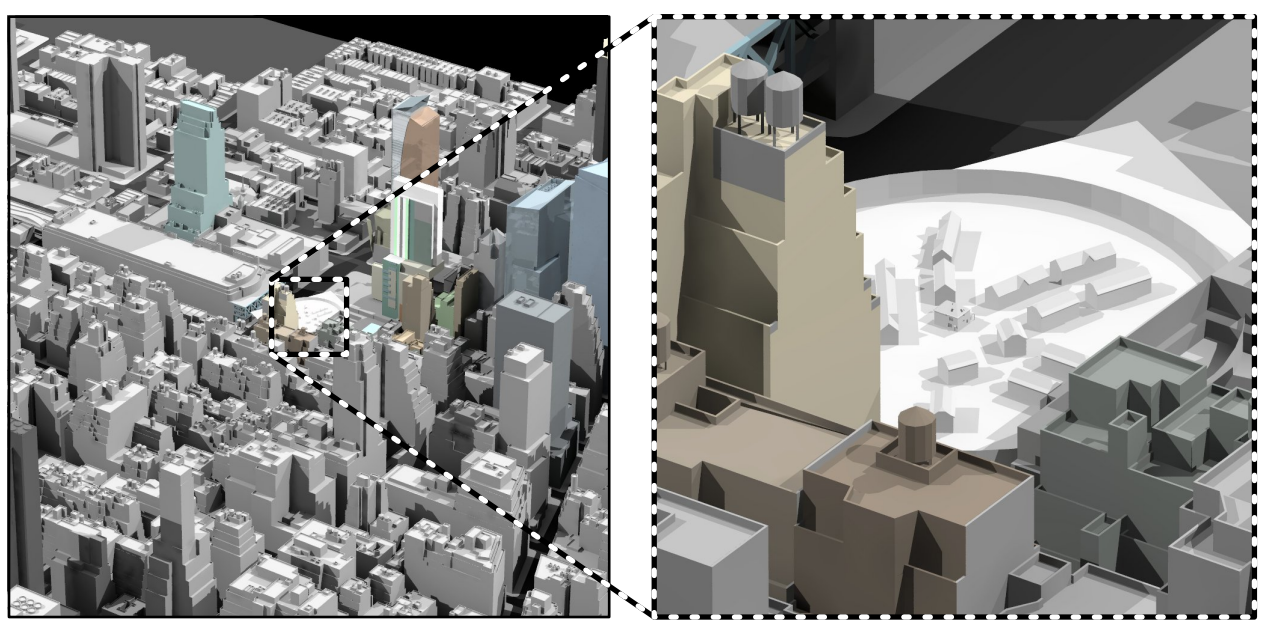

Figure A4: Example application for a highly complex scene 